\title{
Love wave propagation in electro-magneto non-homogeneous elastic media
}

\author{
Shikha Kakar ${ }^{1}$, Rajneesh Kakar ${ }^{2, *}$ \\ ${ }^{1}$ Assistant Professor, SBBSIET, Jalandhar, Punjab-144001, India \\ ${ }^{2}$ Principal, DIPS Polytechnic College, Hoshiarpur-146001, India
}

Email address:

rkakar_163@rediffmail.com (R. Kakar)

\section{To cite this article:}

Shikha Kakar, Rajneesh Kakar. Love Wave Propagation in Electro-Magneto Non-Homogeneous Elastic Media, International Journal of Materials Science and Applications. Vol. 2, No. 2, 2013, pp. 61-67. doi: 10.11648/j.ijmsa.20130202.15

\begin{abstract}
A mathematical approach is taken to investigate Love wave propagation in non-homogeneous electromagneto-elastic media. The elastic meḑia is assumed to be initiąlly, unstressed and at rest. In this study, it is assumed that mass density' $\rho^{\prime}$ ', elastic coefficients ${ }^{\prime} c_{i j}$, dielectric coefficients ${ }^{\prime} \varepsilon_{i j}{ }^{\prime}$ magnetic permeability coefficients ${ }^{\prime} \mu_{i j}{ }^{\prime}$, piezoelectric coefficients ${ }_{i j}$ piezomagnetic coefficients ' $f_{i j}^{\prime}$ and magneto-electric coefficients ' $g_{i j}{ }^{\prime}$ of the specimen are space dependent. The analytical solution for dispersion of love waves is obtained for electromagnetically short and open case. It has been shown that non-homogeneity factor reduces the penetration depth of Love waves. The numerically results are shown graphically.
\end{abstract}

Keywords: Love Waves, Inhomogeneity, Electro-Magneto-Elastic

\section{Introduction}

The electro- magneto-elastic solid material is a class of materials which exhibit the coupling between mechanical, electric and magnetic fields. Composite materials which are made of piezoelectric and piezomagnetic phases show a magneto-electric effect which is not present in individual constituents. These materials show a significant coupling of elastic, magnetic, electric fields due to coupled nature of constitutive equations.

The elastic cylindrical shell under radial impulse was studied by Mcivor [1]. Cinelli [2] has investigated dynamic vibrations and stresses in elastic cylinders and spheres. Pan and Heyliger [3] have given the exact solutions for magneto-electro-elastic Laminates in cylindrical bending. The wave propagation in non-homogeneous magneto-electroelastic plates has been solved by Bin et al. [4]. Kong et al. [5] solved the problem of thermo-magneto-dynamic stresses and perturbation of magnetic field vector in nonhomogeneous hollow cylinder.

A layered structure consisting of an isotropic layer on an isotropic substrate and perfect bonding at their interface was first studied by Love [6]. A Love waves in layered systems consisting of two cubic piezoelectric crystals was studied by Zakharenko [7]. Bracke [8] developed a broadband magneto-electric transducer using a composite ma- terial. Qian et al. [9] have studied Love waves propagation in a piezoelectric layered structure with initial stresses. Later Wang and Quek [10] presented Love waves in piezoelectric coupled solid media. Ramirez et al. [11] studied the free vibration response of two-dimensional magnetoelectro-elastic laminated plates. Aboudi [12] analyzed the micromechanical analysis of fully coupled electromagneto-thermo-elastic multiphase composites. Effect of magnetic bias field on magneto-electric coupling in magneto-electric composites was discussed by Liu [13]. Zaitsev et al. [14] investigated the acoustic waves in piezoelectric viscous and conductive plates. Propagation of Love waves in prestressed piezoelectric layered structures loaded with viscous liquid was studied by Du et al. [15]. Pan [16] presented the exact solution for simply supported and multilayered magneto-electro-elastic plates. The behavior of Love waves in a piezoelectric structure was discussed by Liu [17]. Recently, Kakar [18, 19] has studied the propagation of Love waves in a non-homogeneous elastic media and propagation of Love waves in a non-homogeneous orthotropic layer under compression ' $\mathrm{P}$ ' overlying semiinfinite non-homogeneous medium.

In this study, we consider the wave propagation in nonhomogeneous media, when mass density' $\rho$ ', elastic coefficients ' $c_{i j}$ ', dielectric coefficients ' $\varepsilon_{i j}$ ' magnetic permeability coefficients ${ }^{\prime} \mu_{i j}{ }^{\prime}$, piezoelectric coefficients ' $e_{i j}{ }^{\prime}$ piezo- 
magnetic coefficients ' $f_{i j}$ ' and magneto-electric coefficients ' $g_{i j}$ ' of the specimen space dependent such that the wave velocity is also space dependent and obey the laws $\rho=\rho^{0} e^{2 \alpha x}, c_{i j}=c_{i j}^{0} e^{2 \alpha x}, \varepsilon_{i j}=\varepsilon_{i j}^{0} e^{2 \alpha x}, \mu_{i j}=\mu_{i j}^{0} e^{2 \alpha x}, e_{i j}=e_{i j}^{0} e^{2 \alpha x}$, $f_{i j}=f_{i j}^{0} e^{2 \alpha x}$ and $g_{i j}=g_{i j}^{0} e^{2 \alpha x}$. The specimen is polarized in the $\mathrm{z}$-axis direction. The electric and magnetic potential are calculated and discussed in detail. The paper ends with numerical analysis by taking material parameters. The outcome of these results can be utilized in the design of SAW devices with high performances.

\section{Basic Equations and Formulation of the Problem}

Consider the inhomogeneous transversely isotropic magneto-electro-elastic half space as shown in figure 1 . The piezoelectricity and piezomagneticity are polarized in the $\mathrm{z}$-axis direction. It is assumed, the antiplane displacement components $(u, v, w)$ are given by

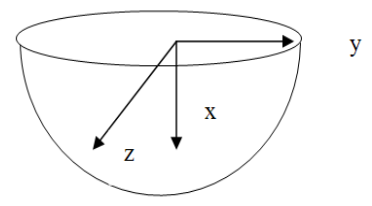

Fig. 1. Geometry of the problem.

$$
\begin{aligned}
& u(x, y)=0, \\
& v(x, y)=0, \\
& w=w(x, y, t),
\end{aligned}
$$
by

The electric and magnetic potential $(\phi$ and $\psi)$ are given

$$
\phi=\phi(x, y, t), \quad \psi=\psi(x, y, t)
$$

The equilibrium equations of elasticity without body forces are given by [20]

$$
\sigma_{i j, j}=\rho \ddot{u}_{i},
$$

The Gauss's laws of electromagnetism without free charges are given by

$$
D_{i, i}=0, B_{i, i}=0
$$

where $\sigma_{i j}, D_{i}$ and $B_{i}$ are the stress, electric displacement and magnetic induction respectively.

The coupled constitutive equations for an anisotropic and linearly magneto-electro-elastic solid can be written as

$$
\begin{aligned}
& \sigma_{i}=c_{i k} \gamma_{k}-e_{k i} E_{k}-f_{k i} H_{k}, \\
& D_{i}=e_{i k} \gamma_{k}+\varepsilon_{k i} E_{k}+g_{k i} H_{k}, \\
& B_{i}=f_{i k} \gamma_{k}+g_{k i} E_{k}+\mu_{k i} H_{k},
\end{aligned}
$$

where ' $c_{i j}$ ', ' $\varepsilon_{i j}$ ' and ' $\mu_{i j}$ ' are elastic coefficients, dielectric coefficients and magnetic permeability coefficients respectively; ' $e_{i j}$ ', ' $f_{i j}$ ' and ' $g_{i j}$ ' are piezoelectric coefficients, piezomagnetic coefficients and magneto-electric coefficients respectively and they are represented by various matrices (for electro-magneto-elastic medium with polling direction) as [21]

$$
\begin{aligned}
& {[\varepsilon]=\left[\begin{array}{ccc}
\varepsilon_{11} & 0 & 0 \\
0 & \varepsilon_{22} & 0 \\
0 & 0 & \varepsilon_{33}
\end{array}\right],} \\
& {[g]=\left[\begin{array}{ccc}
g_{11} & 0 & 0 \\
0 & g_{22} & 0 \\
0 & 0 & g_{33}
\end{array}\right],} \\
& {[\mu]=\left[\begin{array}{ccc}
\mu_{11} & 0 & 0 \\
0 & \mu_{22} & 0 \\
0 & 0 & \mu_{33}
\end{array}\right],}
\end{aligned}
$$

$$
\begin{aligned}
{[c] } & =\left[\begin{array}{cccccc}
c_{11} & c_{12} & c_{13} & 0 & 0 & 0 \\
0 & c_{22} & c_{23} & 0 & 0 & 0 \\
0 & 0 & c_{33} & 0 & 0 & 0 \\
0 & 0 & 0 & c_{44} & 0 & 0 \\
0 & 0 & 0 & 0 & c_{55} & 0 \\
0 & 0 & 0 & 0 & 0 & c_{66}
\end{array}\right], \\
{[e] } & =\left[\begin{array}{ccc}
0 & 0 & e_{31} \\
0 & 0 & e_{32} \\
0 & 0 & e_{33} \\
0 & e_{24} & 0 \\
e_{15} & 0 & 0 \\
0 & 0 & 0
\end{array}\right], \\
{[f] } & =\left[\begin{array}{ccc}
0 & 0 & f_{31} \\
0 & 0 & f_{32} \\
0 & 0 & f_{33} \\
0 & f_{24} & 0 \\
f_{15} & 0 & 0 \\
0 & 0 & 0
\end{array}\right],
\end{aligned}
$$

The stress strain relation is

$$
\begin{aligned}
& \varepsilon_{x}=u_{, x}, \quad \varepsilon_{y}=v_{, y}, \\
& \varepsilon_{z}=w_{, z}, \quad \gamma_{y z}=v_{, z}+w_{, y}, \\
& \gamma_{z x}=u_{, z}+w_{, x}, \quad \gamma_{x y}=v_{, x}+u_{, y},
\end{aligned}
$$

The components of electric and magnetic field are given by

$$
\begin{aligned}
& E_{x}=-\phi_{, x}, E_{y}=-\phi_{, y}, E_{z}=-\psi_{, z}, \\
& H_{x}=-\psi_{, x}, H_{y}=-\psi_{, y}, H_{z}=-\psi_{, z},
\end{aligned}
$$

where $\phi$ and $\psi$ are electric and magnetic potentials.

From Eqs (1)-(5), the coupling wave equations and constitutive equations can be obtained as 


$$
\begin{aligned}
& c_{44}(x) \nabla^{2} w+\frac{\partial c_{44}(x)}{\partial x} \frac{\partial w}{\partial x} \\
& +e_{15}(x) \nabla^{2} \phi+\frac{\partial e_{15}(x)}{\partial x} \frac{\partial \phi}{\partial x} \\
& +f_{15}(x) \nabla^{2} \psi+\frac{\partial f_{15}(x)}{\partial x} \frac{\partial \psi}{\partial x}=\rho(x) \frac{\partial^{2} w}{\partial t^{2}} \\
& e_{15}(x) \nabla^{2} w+\frac{\partial e_{15}(x)}{\partial x} \frac{\partial w}{\partial x} \\
& -\varepsilon_{11}(x) \nabla^{2} \phi-\frac{\partial \varepsilon_{11}(x)}{\partial x} \frac{\partial \phi}{\partial x} \\
& -g_{11}(x) \nabla^{2} \psi-\frac{\partial g_{11}(x)}{\partial x} \frac{\partial \psi}{\partial x}=0 \\
& f_{15}(x) \nabla^{2} w+\frac{\partial f_{15}(x)}{\partial x} \frac{\partial w}{\partial x} \\
& -g_{11}(x) \nabla^{2} \phi-\frac{\partial g_{11}(x)}{\partial x} \frac{\partial \phi}{\partial x} \\
& -\mu_{11}(x) \nabla^{2} \psi-\frac{\partial \mu_{11}(x)}{\partial x} \frac{\partial \psi}{\partial x}=0 .
\end{aligned}
$$

where the nonzero stress, electric displacement and magnetic induction components are

$$
\begin{aligned}
& \sigma_{x z}=c_{44}(x) \frac{\partial w}{\partial x}+e_{15}(x) \frac{\partial \phi}{\partial x}+f_{15}(x) \frac{\partial \psi}{\partial x}, \\
& D_{x}=e_{15}(x) \frac{\partial w}{\partial x}-\varepsilon_{11}(x) \frac{\partial \phi}{\partial x}-g_{11}(x) \frac{\partial \psi}{\partial x}, \\
& B_{x}=f_{15}(x) \frac{\partial w}{\partial x}-g_{11}(x) \frac{\partial \phi}{\partial x}-\mu_{11}(x) \frac{\partial \psi}{\partial x} .
\end{aligned}
$$

Let us assume the following non-homogeneities

$$
\begin{aligned}
& \rho=\rho^{0} e^{2 \alpha x}, c_{44}(x)=c_{44}^{0} e^{2 \alpha x} \\
& , \varepsilon_{11}=\varepsilon_{11}^{0} e^{2 \alpha x}, \\
& \mu_{11}=\mu_{11}^{0} e^{2 \alpha x}, e_{15}=e_{15}^{0} e^{2 \alpha x}, \\
& f_{15}=f_{15}^{0} e^{2 \alpha x}, g_{11}=g_{11}^{0} e^{2 \alpha x} .
\end{aligned}
$$

From Eq. (9) and Eq. (10), we get

$$
\begin{aligned}
& c_{44}^{0}\left(\nabla^{2} w+2 \alpha \frac{\partial w}{\partial x}\right)+e_{15}^{0}\left(\nabla^{2} \phi+2 \alpha \frac{\partial \phi}{\partial x}\right) \\
& +f_{15}^{0}\left(\nabla^{2} \psi+2 \alpha \frac{\partial \psi}{\partial x}\right)=\rho^{0} \frac{\partial^{2} w}{\partial t^{2}}, \\
& e_{15}^{0}\left(\nabla^{2} w+2 \alpha \frac{\partial w}{\partial x}\right)-\varepsilon_{11}^{0}\left(\nabla^{2} \phi-2 \alpha \frac{\partial \phi}{\partial x}\right) \\
& -g_{11}^{0}\left(\nabla^{2} \psi-2 \alpha \frac{\partial \psi}{\partial x}\right)=0, \\
& f_{15}^{0}\left(\nabla^{2} w+2 \alpha \frac{\partial w}{\partial x}\right)-g_{11}^{0}\left(\nabla^{2} \phi-2 \alpha \frac{\partial \phi}{\partial x}\right) \\
& -\mu_{11}^{0}\left(\nabla^{2} \psi-2 \alpha \frac{\partial \psi}{\partial x}\right)=0 .
\end{aligned}
$$

where $\nabla^{2}=\frac{\partial^{2}}{\partial x^{2}}+\frac{\partial^{2}}{\partial z^{2}}$.

Let us assume the upper layer is air then the electric and magnetic potential functions in air are

$$
\phi_{0}=\phi(x, y, t), \quad \psi_{0}=\psi(x, y, t),
$$

Eq. (13) satisfies the Laplace equation

$$
\nabla^{2} \phi_{0}=0, \nabla^{2} \psi_{0}=0
$$

Similarly, the electric displacement and magnetic induction components in the air are

$$
D_{x}^{0}=-\varepsilon_{0} \frac{\partial \phi_{0}}{\partial x}, B_{x}^{0}=-\mu_{0} \frac{\partial \psi_{0}}{\partial x} .
$$

For air, $\mu_{0}=4 \pi \times 10^{-7}$ and $\varepsilon_{0}=8.85 \times 10^{-12}$ (in SI units) known as permeability and permittivity.

\section{Boundary Conditions}

1. For $(x=0)$

$$
\sigma_{x z}(0, y)=0
$$

2. The electric and magnetic potential for electromagnetically open case is (traction free $\operatorname{surface}(x=0)$ ),

$$
\begin{array}{r}
\phi(0, y)=\phi_{0}(0, y), D_{x}(0, y)=D_{x}^{0}(0, y) \\
\psi(0, y)=\psi_{0}(0, y), B_{x}(0, y)=B_{x}^{0}(0, y)
\end{array}
$$

3. For electromagnetically shorted case $(x=0)$

$$
\phi(0, y)=\psi(0, y)=0
$$

4. The attenuation conditions are For

$$
x \rightarrow+\infty, w(x, y), \phi(x, y), \psi(x, y) \rightarrow 0
$$

For

$$
x \rightarrow-\infty, \phi_{0}(x, y), \psi_{0}(x, y) \rightarrow 0
$$

\section{Solution of the Problem}

\subsection{For Electromagnetically Shorted Case}

We may assume the solution of Eq. (12) as

$$
\begin{aligned}
& w=A e^{m x+i k(y-c t)} \\
& \phi=B e^{n x+i k(y-c t)}+\xi A e^{m x+i k(y-c t)} \\
& \psi=C e^{n x+i k(y-c t)}+\zeta A e^{m x+i k(y-c t)}
\end{aligned}
$$

where

$$
\begin{gathered}
m=-\alpha-\sqrt{\alpha^{2}-k\left(\frac{c^{2}}{c_{1}^{2}}-1\right)}, \\
n=-\alpha-\sqrt{\alpha^{2}+k^{2}}, \\
c_{1}=\sqrt{\frac{\bar{c}_{44}^{0}}{\rho}}=\sqrt{\frac{c_{44}^{0}+\xi e_{15}^{0}+\zeta f_{15}^{0}}{\rho}}, \\
\xi=\frac{e_{15}^{0} \mu_{11}^{0}-f_{15}^{0} g_{11}^{0}}{\varepsilon_{11}^{0} \mu_{11}^{0}-g_{11}^{0}}
\end{gathered}
$$

and

$$
\zeta=\frac{f_{15}^{0} \varepsilon_{11}^{0}-e_{15}^{0} g_{11}^{0}}{\varepsilon_{11}^{0} \mu_{11}^{0}-g_{11}^{0}} .
$$

$\mathrm{A}, \mathrm{B}$ and $\mathrm{C}$ are arbitrary constants, $\mathrm{c}$ is phase velocity of 
Love waves, $\mathrm{k}$ is wave number $(k=2 \pi / \lambda)$.

From Eq. (10) and Eq. (22), we get

$$
\begin{aligned}
& \sigma_{x z}=e^{2 \alpha x}\left\{\begin{array}{l}
m\left(c_{44}^{0}+\xi e_{15}^{0}\right. \\
\left.+\zeta f_{15}^{0}\right) A e^{m x+i k(y-c t)} \\
+n e_{15}^{0} B e^{n x+i k(y-c t)} \\
+n f_{15}^{0} C e^{n x+i k(y-c t)}
\end{array}\right\}, \\
& D_{x}=e^{2 \alpha x}\left\{\begin{array}{l}
-n \varepsilon_{11}^{0} B e^{n x+i k(y-c t)} \\
-n g_{11}^{0} C e^{n x+i k(y-c t)}
\end{array}\right\}, \\
& B_{x}=e^{2 \alpha x}\left\{\begin{array}{l}
-n g_{11}^{0} B e^{n x+i k(y-c t)} \\
-n \mu_{11}^{0} C e^{n x+i k(y-c t)}
\end{array}\right\} .
\end{aligned}
$$

Put Eq. (22) and Eq. (23) into the Eq. (16) and Eq. (19), we get

$$
\begin{aligned}
& m\left(c_{44}^{0}+\xi e_{15}^{0}+\zeta f_{15}^{0}\right) A \\
& +n e_{15}^{0} B+n f_{15}^{0} C=0, \\
& B+\xi A=0, \\
& C+\zeta A=0
\end{aligned}
$$

Eliminating A, B and C from Eq. (24), we get the solution for the electromagnetically shorted case

$$
\begin{gathered}
\frac{\alpha+\sqrt{\alpha^{2}-k\left(\frac{c^{2}}{c_{1}^{2}}-1\right)}}{\alpha+\sqrt{\alpha^{2}+k^{2}}} \\
=\left(\frac{\xi e_{15}^{0}+\zeta f_{15}^{0}}{c_{44}^{0}+\xi e_{15}^{0}+\zeta f_{15}^{0}}\right) \\
\frac{m}{n}=k_{0}^{2}
\end{gathered}
$$

where $k_{0}=\sqrt{\frac{\xi e_{15}^{0}+\zeta f_{15}^{0}}{c_{44}^{0}+\xi e_{15}^{0}+\zeta f_{15}^{0}}}$ known as electromagneto-elastic coupling coefficient.

Discussion

It is quite clear that from Eq. (25) Love waves in magneto-electro-elastic half space are dispersive and depend on the non-homogeneity parameters of the media also phase velocity of these waves is related to wave number.

In the absence of non-homogeneity, i.e $\alpha=0$, Eq. (25) reduces to

$$
\frac{c}{c_{1}}=\sqrt{1-k_{0}^{4}}=\sqrt{1-\left(\frac{\xi e_{15}^{0}+\zeta f_{15}^{0}}{c_{44}^{0}+\xi e_{15}^{0}+\zeta f_{15}^{0}}\right)^{2}}
$$

If $f_{15}^{0}=0$ and $g_{11}^{0}=0$, then $\xi=\frac{e_{15}^{0} \mu_{11}^{0}-f_{15}^{0} g_{11}^{0}}{\varepsilon_{11}^{0} \mu_{11}^{0}-g_{11}^{0}}$ and $\zeta=\frac{f_{15}^{0} \varepsilon_{11}^{0}-e_{15}^{0} g_{11}^{0}}{\varepsilon_{11}^{0} \mu_{11}^{0}-g_{11}^{0}}$ reduce to $\xi=\frac{e_{15}^{0}}{\varepsilon_{11}^{0}}$ and $\zeta=0$ respectively. Therefore,

$$
c_{1}=\sqrt{\frac{c_{44}^{0}+\xi e_{15}^{0}+\zeta f_{15}^{0}}{\rho}} \rightarrow c_{1}^{\prime}=\sqrt{\frac{c_{44}^{0}+e_{15}^{0}{ }^{2} / \varepsilon_{11}^{0}}{\rho}}
$$

and

$$
k_{0}=\sqrt{\frac{\xi e_{15}^{0}+\zeta f_{15}^{0}}{c_{44}^{0}+\xi e_{15}^{0}+\zeta f_{15}^{0}}} \rightarrow k_{0}^{\prime}=\sqrt{\frac{e_{15}^{0} / \varepsilon_{11}^{0}}{c_{44}^{0}+e_{15}^{0} / \varepsilon_{11}^{0}}}
$$

Hence, Eq. (25) gives is the wave velocity for piezoelectric non-homogeneous half-space electrically shorted case i.e.

$$
\frac{m}{n}=k_{0}^{\prime 2}
$$

If $\alpha=0$, the phase velocity for piezoelectric homogeneous half-space is given by

$$
\frac{c}{c_{1}^{\prime}}=\sqrt{1-k_{0}^{\prime 4}}=\sqrt{1-\left(\frac{e_{15}^{02} / \varepsilon_{11}^{0}}{c_{44}^{0}+e_{15}^{02} / \varepsilon_{11}^{0}}\right)^{2}}
$$

where, $k_{0}^{\prime}=\sqrt{\frac{e_{15}^{02} / \varepsilon_{11}^{0}}{c_{44}^{0}+e_{15}^{02} / \varepsilon_{11}^{0}}}$

If $e_{15}^{0}=0$ and $g_{11}^{0}=0$, then $\xi=\frac{e_{15}^{0} \mu_{11}^{0}-f_{15}^{0} g_{11}^{0}}{\varepsilon_{11}^{0} \mu_{11}^{0}-g_{11}^{0}}$ and $\zeta=\frac{f_{15}^{0} \varepsilon_{11}^{0}-e_{15}^{0} g_{11}^{0}}{\varepsilon_{11}^{0} \mu_{11}^{0}-g_{11}^{0}}$ reduce to $\xi=0$ and $\zeta=\frac{f_{15}^{0}}{\mu_{11}^{0}}$ respectively. Therefore,

$$
c_{1}=\sqrt{\frac{c_{44}^{0}+\xi e_{15}^{0}+\zeta f_{15}^{0}}{\rho}} \rightarrow c_{1}^{\prime \prime}=\sqrt{\frac{c_{44}^{0}+f_{15}^{02} / \mu_{11}^{0}}{\rho}}
$$

and

$$
k_{0}=\sqrt{\frac{\xi e_{15}^{0}+\zeta f_{15}^{0}}{c_{44}^{0}+\xi e_{15}^{0}+\zeta f_{15}^{0}}} \rightarrow k_{0}{ }_{0}=\sqrt{\frac{f_{15}^{02} / \mu_{11}^{0}}{c_{44}^{0}+f_{15}^{02} / \mu_{11}^{0}}}
$$

Hence, Eq. (25) gives is the wave velocity for piezomagnetic non-homogeneous half-space electrically shorted case i.e.

$$
\frac{m}{n}=k_{0}^{\prime 2}
$$

If $\alpha=0$, the phase velocity for piezomagnetic homogeneous half-space is given by

$$
\frac{c}{c "_{1}}=\sqrt{1-k n_{0}^{4}}=\sqrt{1-\left(\frac{f_{15}^{02} / \mu_{11}^{0}}{c_{44}^{0}+f_{15}^{02} / \mu_{11}^{0}}\right)^{2}}
$$

$$
\text { where, } k "_{0}=\sqrt{\frac{f_{15}^{02} / \mu_{11}^{0}}{c_{44}^{0}+f_{15}^{02} / \mu_{11}^{0}}}
$$

\subsection{For Electromagnetically Open Case}

We may assume the solution of Eq. (14) satisfying the boundary condition Eq. (20) and Eq. (21) are

$$
\begin{aligned}
& \phi_{0}=G e^{k(x+i(y-c t))} \\
& \psi_{0}=H e^{k(x+i(y-c t))}
\end{aligned}
$$

The solution of Eq. (15) satisfying the boundary condi- 
tion Eq. (20) and Eq. (21) are

$$
\begin{aligned}
& D_{x}^{0}=-\varepsilon_{0} k G e^{k(x+i(y-c t))} \\
& B_{x}^{0}=-\mu_{0} k H e^{k(x+i(y-c t))}
\end{aligned}
$$

Put Eq. (22) and Eq. (23) into the Eq. (17) and Eq. (18), we get

$$
\begin{gathered}
m\left(c_{44}^{0}+\xi e_{15}^{0}+\zeta f_{15}^{0}\right) A \\
+n e_{15}^{0} B+n f_{15}^{0} C=0, \\
B+\xi A=G, \\
C+\zeta A=H \\
\varepsilon_{11}^{0} n B+g_{11}^{0} n C=\varepsilon_{0} k G \\
g_{11}^{0} n B+\mu_{11}^{0} n C=\mu_{0} k H
\end{gathered}
$$

Eliminating A, B, C, G and H from Eq. (33), we get the solution for the electromagnetically open case

$$
\begin{aligned}
& \left(\begin{array}{l}
\varepsilon_{11}^{0} n-\varepsilon_{0} k+ \\
\frac{\xi \varepsilon_{0} k n e_{15}^{0}}{m\left(c_{44}^{0}+\xi e_{15}^{0}+\zeta f_{15}^{0}\right)}
\end{array}\right)\left(\begin{array}{l}
\mu_{11}^{0} n-\mu_{0} k \\
+\frac{\zeta \mu_{0} k n f_{15}^{0}}{m\left(c_{44}^{0}+\xi e_{15}^{0}+\zeta f_{15}^{0}\right)}
\end{array}\right) \\
& -n^{2}\left(\begin{array}{l}
g_{11}^{0} n \\
+\frac{\zeta \mu_{0} k e_{15}^{0}}{m\left(c_{44}^{0}+\xi e_{15}^{0}+\zeta f_{15}^{0}\right)}
\end{array}\right)=0
\end{aligned}
$$

\section{Discussion}

It is quite clear that from Eq. (34) Love waves in magneto-electro-elastic half space are dispersive and depend on the non-homogeneity parameters of the media also phase velocity of these waves is related to wave number.

In the absence of non-homogeneity, i.e $\alpha=0$, Eq. (25) reduces to

$$
\frac{c}{c_{2}}=\sqrt{1-\tau_{0}^{2}}
$$

where

$$
\begin{aligned}
& \xi \varepsilon_{0} e_{15}^{0}\left(\mu_{11}^{0}+\mu_{0}\right) \\
& +\zeta \mu_{0} f_{15}^{0}\left(\varepsilon_{11}^{0}+\varepsilon_{0}\right) \\
& \tau=\frac{+g_{11}^{0}\left(\zeta e_{15}^{0} \mu_{11}^{0}+\xi \varepsilon_{0} f_{15}^{0}\right)}{\left(c_{44}^{0}+e_{15}^{02} / \varepsilon_{11}^{0}\right)\left(\left(\varepsilon_{11}^{0}+\varepsilon_{0}\right)\left(\mu_{11}^{0}+\mu_{0}\right)-g_{11}^{0}\right)}
\end{aligned}
$$

If $f_{15}^{0}=0$ and $g_{11}^{0}=0$, then $\xi=\frac{e_{15}^{0} \mu_{11}^{0}-f_{15}^{0} g_{11}^{0}}{\varepsilon_{11}^{0} \mu_{11}^{0}-g_{11}^{0}}$ and $\zeta=\frac{f_{15}^{0} \varepsilon_{11}^{0}-e_{15}^{0} g_{11}^{0}}{\varepsilon_{11}^{0} \mu_{11}^{0}-g_{11}^{0}}$ reduce to $\xi=\frac{e_{15}^{0}}{\varepsilon_{11}^{0}}$ and $\zeta=0$ respectively. Therefore,

$$
\varepsilon_{11}^{0} n-\varepsilon_{0} k+\frac{\varepsilon_{0} k n}{m} k_{0}^{\prime 2}=0
$$

If $\alpha=0$, the phase velocity for piezoelectric homogeneous half-space is given by

$$
\frac{c}{c^{\prime}{ }_{2}}=\sqrt{1-\frac{k_{0}^{\prime 4}}{\left(1+\frac{\varepsilon_{11}^{0}}{\varepsilon_{0}}\right)^{2}}}
$$

where, $k_{0}{ }_{0}=\sqrt{\frac{e_{15}^{0}{ }^{2} / \varepsilon_{11}^{0}}{c_{44}^{0}+e_{15}^{0}{ }^{2} / \varepsilon_{11}^{0}}}$

If $e_{15}^{0}=0$ and $g_{11}^{0}=0$, then $\xi=\frac{e_{15}^{0} \mu_{11}^{0}-f_{15}^{0} g_{11}^{0}}{\varepsilon_{11}^{0} \mu_{11}^{0}-g_{11}^{0}}$ and $\zeta=\frac{f_{15}^{0} \varepsilon_{11}^{0}-e_{15}^{0} g_{11}^{0}}{\varepsilon_{11}^{0} \mu_{11}^{0}-g_{11}^{0}}$ reduce to $\xi=0$ and $\zeta=\frac{f_{15}^{0}}{\mu_{11}^{0}}$ respectively.

Therefore,

$$
\mu_{11}^{0} n-\mu_{0} k+\frac{\mu_{0} k n}{m} k_{0}^{\prime 2}=0
$$

If $\alpha=0$, the phase velocity for piezomagnetic homogeneous half-space is given by

$$
\frac{c}{c^{\prime \prime}}=\sqrt{1-\frac{k_{0}^{n_{0}^{4}}}{\left(1+\frac{\mu_{11}^{0}}{\mu_{0}}\right)^{2}}}
$$

where, $k "_{0}=\sqrt{\frac{f_{15}^{02} / \mu_{11}^{0}}{c_{44}^{0}+f_{15}^{02} / \mu_{11}^{0}}}$.

\section{Numerical Analysis}

To study the propagation behavior of Love waves in non-homogeneous electro-magneto-half- space, the following parameters are taken as shown in table-1. Using these

\begin{tabular}{|c|c|c|c|c|c|c|}
\hline$c_{44}^{0}$ & $e_{15}^{0}$ & $f_{15}^{0}$ & $g_{11}^{0}$ & $\mu_{11}^{0}$ & $\varepsilon_{11}^{0}$ & $\rho^{0}$ \\
\hline $\begin{array}{l}3 \\
\mathrm{HPa}\end{array}$ & $\begin{array}{l}11.6 \\
\mathrm{C} / \mathrm{m}^{2}\end{array}$ & $\begin{array}{l}550 \\
\text { N/Am }\end{array}$ & $\begin{array}{l}5 \times 10^{-} \\
{ }^{12} \mathrm{Ns} / \mathrm{Vc}\end{array}$ & $\begin{array}{l}5 \times 10^{-} \\
{ }^{5} \mathrm{Ns}^{2} / \mathrm{C}^{2}\end{array}$ & $\begin{array}{l}11.2 \times 10^{-} \\
{ }^{9} \mathrm{~F} / \mathrm{m}\end{array}$ & $\begin{array}{l}7500 \\
\mathrm{Kg} / \mathrm{m}^{3}\end{array}$ \\
\hline
\end{tabular}
parameters we can get the velocity of love waves in the homogeneous electro-magneto-elastic half-space for electrically shorted case from Eq. (28) and it is $2725.698 \mathrm{~m} / \mathrm{s}$. The velocity of love waves in the homogeneous electromagneto-elastic half-space for electrically open case is obtained from Eq. (37) and it comes out to be 2853.398 $\mathrm{m} / \mathrm{s}$.

The variation of non-dimensionless phase velocity $\mathrm{v} / \mathrm{s}$ non-dimensional wave number is also plotted in figure- 2 and in figure-3 with the help of MATLAB. It is important to note that in case of electromagnetically shorted case the non-dimensionally phase velocity started from 0.68 where as for electromagnetically open case the non-dimensionally phase velocity started from 0.88 for Love waves. Also, the effect of gradient coefficient on dispersion curve is more sensitive for short condition than open condition.

Table 1. 


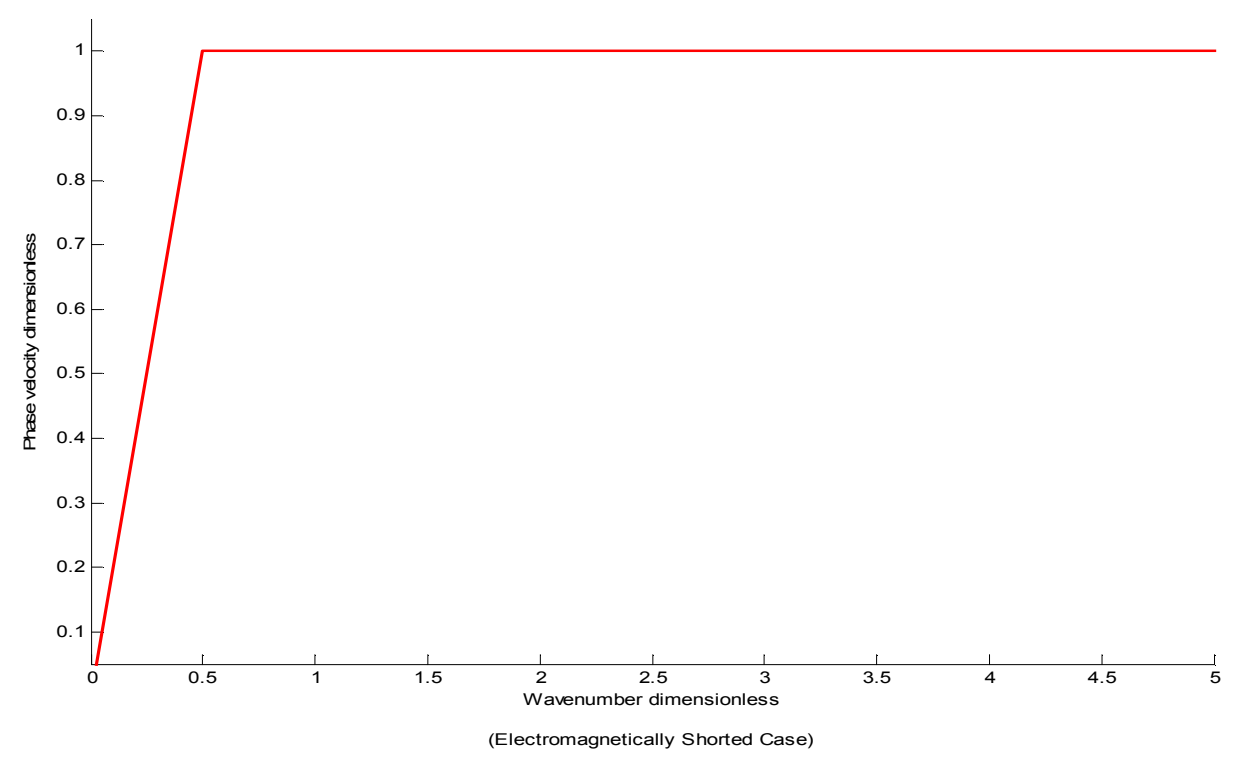

Fig. 2.

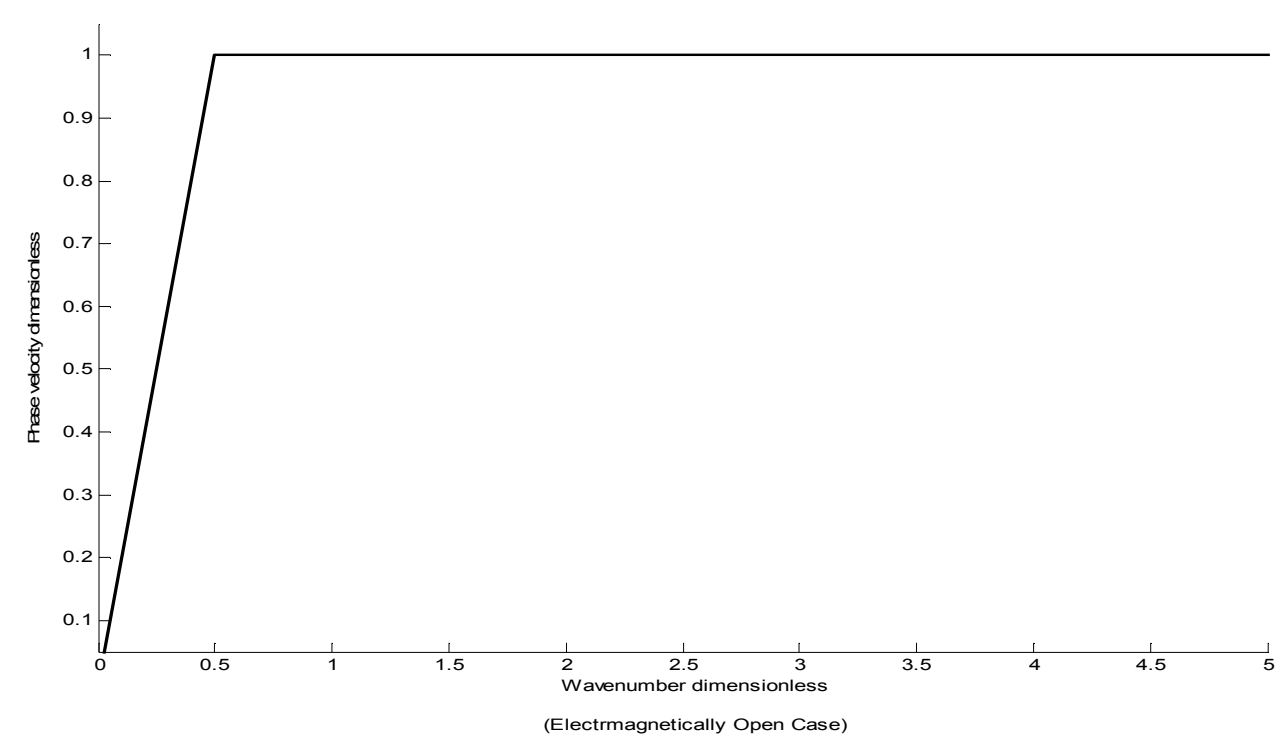

Fig. 3.

\section{Conclusion}

In this study, we have taken into account the effect of non-homogeneity on the propagation of behavior of Love waves in a transversely isotropic electro-magneto-elastic half space. One numerical example is taken to explain the dispersion of Love waves. The following points have been noticed:

The Love waves are dispersive in nature for both electromagnetically open and short cases. These waves are different in non-homogeneous substrate as compared to homogeneous substrate.

The phase velocity is more in electrically open case as compared to shorted case.

The electro-magneto-elastic coupling factor of Love waves can be improved by adapting the appropriate value of the graded coefficient.

The dependence of phase velocity on the inhomogeneity in space for electro-magneto-elastic substrate for Love waves opens a new window for designing acoustic wave electro-magneto-elastic devices.

\section{Acknowledgements}

The authors convey their sincere thanks to DIPS Polytechnic College for facilitating us with best facility. The authors are also thankful to the referees for their valuable comments.

\section{References}


[1] Mcivor I K, 1966, The elastic cylindrical shell under radial impulse, ASME J. Appl. Mech., 33, pp. 831-837.

[2] Cinelli G, 1966, Dynamic vibrations and stresses in elastic cylinders and spheres, ASME J. Appl. Mech., 33, pp. 82530.

[3] Pan E, Heyliger P R, 2005, Exact solutions for Magnetoelectro-elastic Laminates in cylindrical bending, Int. J. of Solid and Struct., 40, pp. 6859-6876.

[4] Bin W, Jiangong Y, 2005, Cunfu H, Wave propagation in non-homogeneous magneto-electro-elastic plates, J. of Sound and Vib., 317, pp. 250-264.

[5] Kong T, Li D X, Wang X, 2009, Thermo-magneto-dynamic stresses and perturbation of magnetic field vector in nonhomogeneous hollow cylinder, Appl. Mathematical Modeling, 33, pp. 2939-2950.

[6] Love A E H, Some Problems of Geodynamics, Cambridge University Press, London, 1911.

[7] Zakharenko A, 2005, A Love-type waves in layered systems consisting of two cubic piezoelectric crystals, J Sound Vibr, 285 , pp. $877-886$.

[8] Bracke L P M, Vliet T G, 1981, A broadband magnetoelectric transducer using a composite materials, Int J Electr, 51, pp. 255-262.

[9] Qian Z, Jin F, Wang Z, 2004, Love waves propagation in a piezoelectric layered structure with initial stresses, Acta Mechanica, 171, pp. 41-57.

[10] Wang Q, Quek S T, 2001, Love waves in piezoelectric coupled solid media. Smart Mater Struct, 10, pp. 380-388.

[11] Ramirez F, Heyliger P R, Pan E, 2006, Free vibration response of two-dimensional magneto-electro-elastic laminated plates, J Sound Vibr, 292, pp. 626-644.
[12] Aboudi J, 2001, Micromechanical analysis of fully coupled electro-magneto-thermo-elastic multiphase composites, Smart Mater. Struct., 10, pp. 867-877.

[13] Liu YX, Wan JG, Liu JM, Nan CW, 2003, Effect of magnetic bias field on magnetoelectric coupling in magnetoelectric composites, J. Appl. Phys., 94, pp.5118-5122.

[14] Zaitsev BD, Kuznetsova IE, Joshi SG, Borodina IA, 2001, Acoustic waves in piezoelectric plates bordered with viscous and conductive liquid, Ultrasonics, 39(1), pp. 45-50.

[15] Du JK, Xian K, Wang J, Yong YK, 2008, Propagation of Love waves in prestressed piezoelectric layered structures loaded with viscous liquid, Acta Mechanica Solida Sinica, 21(6), pp. 542-548.

[16] Pan E, 2001, Exact solution for simply supported and multilayered magneto-electro-elastic plates, ASME J. Appl. Mech. 68, pp. 608-618.

[17] Liu J, Wang ZK, 2005, The propagation behavior of Love waves in a functionally graded layered piezoelectric structure, Smart Mat. Struct., 14, pp. 137-146.

[18] Kakar R, Kakar S, 2012, Propagation of Love Waves in a Non-Homogeneous Elastic Media, J. Acad. Indus. Res., 1(6), pp. 323-328.

[19] Kakar R, Gupta KC, 2012, Propagation of Love Waves in A Non-Homogeneous Orthotropic Layer Under Compression 'P' Overlying Semi-Infinite Non-Homogeneous Medium, Global Journal of Pure and Applied Mathematics, 8(4), pp. 483-494.

[20] Karl F G. Wave Motion in Elastic Solids, Clarendon Press, Oxford, 1975.

[21] Du J, Jin X, Wang J, Ningbo, 2007, Love wave propagation in layered magneto-electro-elastic structures with initial stress Acta Mechanica, 192, pp. 169-189. 